\title{
Correction to: The ESCRT Complexes
}

\section{Emmanuel Culetto and Renaud Legouis}

Correction to:

Emmanuel Culetto and Renaud Legouis (eds.), The ESCRT Complexes:

Methods and Protocols, Methods in Molecular Biology, vol. 1998, https://doi.org/10.1007/978-1-4939-9492-2

This book was inadvertently published with incorrect affiliations for both the editors. Earlier it was:

Emmanuel Culetto and Renaud Legouis

Diabetes and Islet Biology Group, Faculty of Medicine and Health,

NHMRC Clinical Trials Centre, The University of Sydney, Camperdown, NSW, Australia

This has now been amended throughout the book to the following correct affiliation:

Emmanuel Culetto and Renaud Legouis

Institute for Integrative Biology of the Cell (I2BC), CEA, CNRS, Univ.Paris-Sud,

Université Paris-Saclay, Gif-sur-Yvette, France

The updated online version of the book can be found at

https://doi.org/10.1007/978-1-4939-9492-2

https://doi.org/10.1007/978-1-4939-9492-2_4

Emmanuel Culetto and Renaud Legouis (eds.), The ESCRT Complexes: Methods and Protocols, Methods in Molecular Biology, vol. 1998, https://doi.org/10.1007/978-1-4939-9492-2_23, ( ) Springer Science+Business Media, LLC, part of Springer Nature 2019 